\title{
POSITIVE SOLUTIONS FOR HIGHER-ORDER MULTI-POINT FRACTIONAL BOUNDARY VALUE PROBLEMS
}

\author{
İSMAİL YASLAN AND MUSTAFA GÜNENDİ
}

Received 24 September, 2020

\begin{abstract}
In this paper, we consider the higher-order multi-point fractional boundary value problem. We establish the criteria for the existence of at least one and three positive solutions for higher order nonlinear $m$-point fractional boundary value problem by using the Krasnosel'skii fixed point theorem and the Legget-Williams fixed point theorem, respectively.
\end{abstract}

2010 Mathematics Subject Classification: 34B07; 34D05; 34L20; 34K37

Keywords: boundary value problems, cone, fixed point theorems, positive solutions, RiemannLiouville fractional derivative

\section{INTRODUCTION}

Fractional calculus is a generalization of ordinary differentiation and integration to arbitrary (non-integer) order. Fractional differential equations arise in many engineering and scientific disciplines as the mathematical models of systems and processes in the fields of physics, chemistry, aerodynamics, electrodynamics of complex medium, polymer rheology, [1, 2, 6, 7, 13,17-21].

Among all the researches on the theory of the fractional differential equations, the study of the boundary value problems for fractional differential equations recently has attracted a great deal of attention from many researchers. Some results have been obtained on the existence of positive solutions of the boundary value problems for some specific fractional differential equations [3-5, 8-11, 15, 22, 23].

In [16], Nyamoradi and Javidi were interested in the fractional order multi-point boundary value problem

$$
\left\{\begin{array}{l}
D_{0^{+}}^{\sigma}\left(\phi_{p}\left(u^{\prime \prime}(t)\right)\right)-g(t) f(u(t))=0, t \in[0,1], 1<\sigma \leq 2, \\
\phi_{p}\left(u^{\prime \prime}(0)\right)=\phi_{p}\left(u^{\prime \prime}(1)\right)=0 \\
a u(0)-b u^{\prime}(0)=\sum_{i=1}^{m-2} a_{i} u\left(\xi_{i}\right), \\
c u(1)+d u^{\prime}(1)=\sum_{i=1}^{m-2} b_{i} u\left(\xi_{i}\right)
\end{array}\right.
$$


where $D_{0^{+}}^{\sigma}$ is the standard Riemann-Liouville fractional derivative of order $\sigma$. Some existence results for at least one positive solutions were established by using Krasnosel'skii fixed point theorem.

In this paper, we study the existence of positive solutions to multi-point boundary value problem (BVP) for higher order fractional differential equations:

$$
\left\{\begin{array}{l}
-D_{0^{+}}^{\eta-2}\left(u^{\prime \prime}(t)\right)+f(t, u(t))=0, t \in[0,1], \\
u^{\prime \prime}(0)=u^{\prime \prime \prime}(0)=\ldots=u^{(n-2)}(0)=0, u^{\prime \prime \prime}(1)=0, \\
\alpha u(0)-\beta u^{\prime}(0)=\sum_{p=1}^{m-2} a_{p} u^{\prime}\left(\xi_{p}\right), \\
\gamma u(1)+\delta u^{\prime}(1)=\sum_{p=1}^{m-2} b_{p} u^{\prime}\left(\xi_{p}\right),
\end{array}\right.
$$

where $D_{0^{+}}^{\eta-2}$ is the Riemann-Liouville fractional derivative of order $\eta-2$. Throughout the paper, we suppose that $m, n \geq 3$ and $n-1<\eta \leq n$, where $n, m \in \mathbb{N}$ and $\beta>\alpha>1, \gamma, \delta>0, a_{p}, b_{p} \geq 0$ are given constants and $0<\xi_{1}<\ldots<\xi_{m-2}<1$. We assume that $f:[0,1] \times[0, \infty) \rightarrow[0, \infty)$ is continuous.

We have organized the paper as follows. First, we provide some definitions and preliminary lemmas which are key tools for our main results. Second, we obtained the existence of at least one positive solution for the BVP (1.1) by using the Krasnosel'skii fixed point theorem. Finally, we use the Legget-Williams fixed-point theorem to show that the existence of at least three positive solutions to the BVP (1.1).

We assume that the following conditions are satisfied:

$$
\begin{aligned}
& \text { (H1) If } m \geq 3 \text {, then } \gamma \sum_{k=1}^{m-2} a_{k} \geq \alpha \sum_{k=1}^{m-2} b_{k} \text { and } \\
& \text { if } m>3 \text {, then } \alpha \delta>\gamma \sum_{k=1}^{j-1} a_{k} \geq \alpha \sum_{k=1}^{j-1} b_{k}>\beta \gamma \text {, where } 2 \leq j \leq m-2 . \\
& \text { (H2) } \alpha \delta>\alpha \sum_{p=1}^{m-2} b_{p}+\gamma \sum_{p=1}^{m-2} a_{p} .
\end{aligned}
$$

\section{PReliminaries}

To state the main results of this paper, we will need the following lemmas and we present some notation.

Definition 1. The Riemann-Liouville fractional derivative of order $\alpha>0$ for a function $u:(0, \infty) \rightarrow \mathbb{R}$ is defined by

$$
D_{0^{+}}^{\alpha} u(t)=\frac{1}{\Gamma(n-\alpha)} \frac{d^{n}}{d t^{n}} \int_{0}^{t}(t-s)^{n-\alpha-1} u(s) d s,
$$

where $n=[\alpha]+1$. 
Definition 2. The Riemann-Liouville fractional integral of order $\alpha>0$ of a function $u:(0, \infty) \rightarrow \mathbb{R}$ is given by

$$
I_{0^{+}}^{\alpha} u(t)=\frac{1}{\Gamma(\alpha)} \int_{0}^{t}(t-s)^{\alpha-1} u(s) d s,
$$

where $\Gamma(\cdot)$ is the Euler gamma function.

Lemma 1 ([13]). The equality $D_{0^{+}}^{\gamma} I_{0^{+}}^{\gamma} f(t)=f(t), \gamma>0$ holds for $f \in L(0,1)$.

Lemma 2 ([13]). Let $\alpha>0$. Then the differential equation

$$
D_{0^{+}}^{\alpha} u=0
$$

has a unique solution $u(t)=c_{1} t^{\alpha-1}+c_{2} t^{\alpha-2}+\ldots+c_{n} t^{\alpha-n}, c_{i} \in \mathbb{R}, i=1, \ldots, n$, where $n-1<\alpha \leq n$.

Lemma 3 ([13]). Let $\alpha>0$. Then the following equality holds for $u \in L(0,1)$, $D_{0^{+}}^{\alpha} u \in L(0,1)$ :

$$
I_{0^{+}}^{\alpha} D_{0^{+}}^{\alpha} u(t)=u(t)+c_{1} t^{\alpha-1}+c_{2} t^{\alpha-2}+\ldots+c_{n} t^{\alpha-n},
$$

$c_{i} \in \mathbb{R}, i=1, \ldots, n$, where $n-1<\alpha \leq n$.

If $-u^{\prime \prime}(t)=y(t)$ and $\eta-2=\sigma$, then the problem

$$
\left\{\begin{array}{l}
-D_{0^{+}}^{\eta-2}\left(u^{\prime \prime}(t)\right)+f(t, u(t))=0, t \in[0,1], \\
u^{\prime \prime}(0)=u^{\prime \prime \prime}(0)=\ldots=u^{(n-2)}(0)=0, u^{\prime \prime \prime}(1)=0
\end{array}\right.
$$

is turned into the problem

$$
\left\{\begin{array}{l}
D_{0^{+}}^{\sigma} y(t)+f(t, u(t))=0, t \in[0,1], \\
y(0)=y^{\prime}(0)=\ldots=y^{(n-4)}(0)=0, y^{\prime}(1)=0 .
\end{array}\right.
$$

Lemma 4. The boundary value problem (2.4) has a unique solution

$$
y(t)=\int_{0}^{1} H(t, s) f(s, u(s)) d s,
$$

where

$$
H(t, s)= \begin{cases}\frac{(1-s)^{\sigma-2} t^{\sigma-1}}{\Gamma(\sigma)}, & t \leq s, \\ \frac{(1-s)^{\sigma-2} t^{\sigma-1}-(t-s)^{\sigma-1}}{\Gamma(\sigma)}, & t \geq s .\end{cases}
$$


Proof. According to Lemma 3, we obtain

$$
y(t)=-\frac{1}{\Gamma(\sigma)} \int_{0}^{t}(t-s)^{\sigma-1} f(s, u(s)) d s+c_{1} t^{\sigma-1}+c_{2} t^{\sigma-2}+\ldots+c_{n-2} t^{\sigma-n+2} .
$$

By boundary conditions of (2.4) we get $c_{2}=c_{3}=\ldots=c_{n-2}=0$ and

$$
c_{1}=\frac{1}{\Gamma(\sigma)} \int_{0}^{1}(1-s)^{\sigma-2} f(s, u(s)) d s \text {. }
$$

Thus, the unique solution of problem (2.4) is

$$
\begin{aligned}
y(t) & =\int_{0}^{t} \frac{(1-s)^{\sigma-2} t^{\sigma-1}-(t-s)^{\sigma-1}}{\Gamma(\sigma)} f(s, u(s)) d s+\int_{t}^{1} \frac{(1-s)^{\sigma-2} t^{\sigma-1}}{\Gamma(\sigma)} f(s, u(s)) d s \\
& =\int_{0}^{1} H(t, s) f(s, u(s)) d s .
\end{aligned}
$$

The proof is complete.

Lemma 5. If $(\mathrm{H} 1)$ and $(\mathrm{H} 2)$ hold and

$$
K:=\alpha \gamma+\alpha \delta-\alpha \sum_{p=1}^{m-2} b_{p}+\gamma \beta+\gamma \sum_{p=1}^{m-2} a_{p}
$$

then for $y \in C[0,1]$, the boundary value problem

$$
\left\{\begin{array}{l}
-u^{\prime \prime}(t)=y(t), t \in[0,1] \\
\alpha u(0)-\beta u^{\prime}(0)=\sum_{p=1}^{m-2} a_{p} u^{\prime}\left(\xi_{p}\right) \\
\gamma u(1)+\delta u^{\prime}(1)=\sum_{p=1}^{m-2} b_{p} u^{\prime}\left(\xi_{p}\right)
\end{array}\right.
$$

has a unique solution

$$
u(t)=\int_{0}^{1} G(t, s) y(s) d s
$$


where

$$
G(t, s)=\frac{1}{K}\left\{\begin{array}{r}
(\alpha s+\beta)\left(\gamma(1-t)+\delta-\sum_{p=1}^{m-2} b_{p}\right), 0 \leq s \leq \xi_{1}, t \geq s \\
\left(\gamma(1-s)+\delta-\sum_{p=1}^{m-2} b_{p}\right)(\alpha t+\beta)+\gamma \sum_{p=1}^{m-2} a_{p}(t-s), 0 \leq s \leq \xi_{1}, t \leq s \\
\left(\alpha s+\beta+\sum_{k=1}^{j-1} a_{k}\right)\left(\gamma(1-t)+\delta-\sum_{p=j}^{m-2} b_{p}\right)+\sum_{k=1}^{j-1} b_{k}\left(\alpha(t-s)+\sum_{p=j}^{m-2} a_{p}\right), \\
\xi_{j-1}<s \leq \xi_{j}, t \geq s, 2 \leq j \leq m-2 \\
\left(\gamma(1-s)+\delta-\sum_{p=j}^{m-2} b_{p}\right)\left(\alpha t+\beta+\sum_{k=1}^{j-1} a_{k}\right)+\sum_{p=j}^{m-2} a_{p}\left(\gamma(t-s)+\sum_{k=1}^{j-1} b_{k}\right), \\
\xi_{j-1}<s \leq \xi_{j}, t \leq s, 2 \leq j \leq m-2 \\
\left(\alpha s+\beta+\sum_{k=1}^{m-2} a_{k}\right)(\gamma(1-t)+\delta)+\alpha \sum_{k=1}^{m-2} b_{k}(t-s), \xi_{m-2}<s \leq 1, t \geq s \\
(\gamma(1-s)+\delta)\left(\alpha t+\beta+\sum_{k=1}^{m-2} a_{k}\right), \xi_{m-2}<s \leq 1, t \leq s .
\end{array}\right.
$$

Proof. A direct calculation gives that if $y \in C[0,1]$, then the boundary value problem (2.8) has the unique solution

$$
\begin{aligned}
u(t)= & -\int_{0}^{t}(t-s) y(s) \Delta s+\frac{t}{K}\left\{\alpha \int_{0}^{1}(\gamma(1-s)+\delta) y(s) \Delta s+\sum_{p=1}^{m-2}\left(\gamma a_{p}-\alpha b_{p}\right) \int_{0}^{\xi_{p}} y(s) \Delta s\right\} \\
& +\frac{1}{K}\left\{\left(\beta+\sum_{p=1}^{m-2} a_{p}\right) \int_{0}^{1}(\gamma(1-s)+\delta) y(s) \Delta s+\left(-\left(\beta+\sum_{p=1}^{m-2} a_{p}\right)\right) \sum_{p=1}^{m-2} b_{p} \int_{0}^{\xi_{p}} y(s) \Delta s\right. \\
& \left.+\left(\frac{\gamma\left(\beta+\sum_{p=1}^{m-2} a_{p}\right)}{\alpha}-\frac{K}{\alpha}\right) \sum_{p=1}^{m-2} a_{p} \int_{0}^{\xi_{p}} y(s) \Delta s\right\} .
\end{aligned}
$$

Hence, we obtain (2.9).

Lemma 6. The Green's function $G(t, s)$ in (2.10) satisfies

$$
0<G(t, s) \leq G(s, s)
$$

for $(t, s) \in[0,1] \times[0,1]$. 
Proof. From (H1), (H2) and (2.10), $G(t, s)>0$. Now, we will show that $G(t, s) \leq$ $G(s, s)$.

(i) Let $s \in\left[0, \xi_{1}\right]$ and $t \geq s$. Since $G(t, s)$ is decreasing in $t$, we get $G(t, s) \leq$ $G(s, s)$.

(ii) Let $s \in\left[0, \xi_{1}\right]$ and $t \leq s$. Since $G(t, s)$ is increasing in $t$, we have $G(t, s) \leq$ $G(s, s)$.

(iii) Take $s \in\left(\xi_{j-1}, \xi_{j}\right], 2 \leq j \leq m-2$ and $t \geq s$. From (H1), $G(t, s)$ is decreasing in $t$. So we obtain $G(t, s) \leq G(s, s)$.

(iv) Take $s \in\left(\xi_{j-1}, \xi_{j}\right], 2 \leq j \leq m-2$ and $t \leq s$. Since $G(t, s)$ is increasing in $t$, we get $G(t, s) \leq G(s, s)$.

(v) Let $s \in\left(\xi_{m-2}, 1\right]$ and $t \geq s$. From (H1), $G(t, s)$ is decreasing in $t$. So we have $G(t, s) \leq G(s, s)$.

(vi) Let $s \in\left(\xi_{m-2}, 1\right]$ and $t \leq s$. Since $G(t, s)$ is increasing in $t$, we obtain $G(t, s) \leq$ $G(s, s)$.

Lemma 7. Green's function $G(t, s)$ in (2.10) satisfies

$$
\min _{t \in[0,1]} G(t, s) \geq z\|G(., s)\|
$$

with

$$
z=\min \left\{z_{1}, z_{2}, z_{3}, z_{4}, z_{5}, z_{6}\right\}
$$

where

$$
\begin{aligned}
& z_{1}=\frac{\delta-\sum_{p=1}^{m-2} b_{p}}{\gamma+\delta-\sum_{p=1}^{m-2} b_{p}}, \quad z_{2}=\frac{\left(\alpha \delta-\alpha \sum_{p=1}^{m-2} b_{p}-\gamma \sum_{p=1}^{m-2} a_{p}\right)}{\left(\gamma+\delta-\sum_{p=1}^{m-2} b_{p}\right)(\alpha+\beta)}, \quad z_{3}=\frac{\delta-\sum_{p=j}^{m-2} b_{p}}{\gamma+\delta-\sum_{p=j}^{m-2} b_{p}}, \\
& z_{4}=\frac{\frac{\beta}{\alpha}-1}{\alpha+\beta+\sum_{k=1}^{j-1} a_{k}}, \quad z_{5}=\frac{\delta}{\gamma+\delta}, \quad z_{6}=\frac{\frac{\beta}{\alpha}-1}{\alpha+\beta+\sum_{k=1}^{m-2} a_{k}}
\end{aligned}
$$

and $\|$.$\| is defined by \|x\|=\max _{t \in[0,1]}|x(t)|$.

Proof. (i) Take $s \in\left[0, \xi_{1}\right]$ and $t \geq s$. Since $G(t, s)$ is decreasing in $t$ and $0<z_{1}<1$, we get $\min _{t \in[0,1]} G(t, s)=G(1, s)$ and $\min _{t \in[0,1]} G(t, s) \geq z_{1} G(s, s)=$ $z_{1}\|G(., s)\|$.

(ii) Take $s \in\left[0, \xi_{1}\right]$ and $t \leq s$. Since $G(t, s)$ is increasing in $t$ and $0<z_{2}<1$, we have $\min _{t \in[0,1]} G(t, s)=G(0, s)$ and $\min _{t \in[0,1]} G(t, s) \geq z_{2} G(s, s)=z_{2}\|G(., s)\|$. 
(iii) Let $s \in\left(\xi_{j-1}, \xi_{j}\right], 2 \leq j \leq m-2$ and $t \geq s$. From (H1), $G(t, s)$ is decreasing in $t$. It is clear that $0<z_{3}<1$. So $\min _{t \in[0,1]} G(t, s)=G(1, s)$ and $\min _{t \in[0,1]} G(t, s) \geq$ $z_{3} G(s, s)=z_{3}\|G(., s)\|$.

(iv) Let $s \in\left(\xi_{j-1}, \xi_{j}\right], 2 \leq j \leq m-2$ and $t \leq s$. Since $G(t, s)$ is increasing in $t$ and $0<z_{4}<1$, we have $\min _{t \in[0,1]} G(t, s)=G(0, s)$ and $\min _{t \in[0,1]} G(t, s) \geq z_{4} G(s, s)=$ $z_{4}\|G(., s)\|$.

(v) Take $s \in\left(\xi_{m-2}, 1\right]$ and $t \geq s$. From (H1), $G(t, s)$ is decreasing in $t$. It is clear that $0<z_{5}<1$. So we get $\min _{t \in[0,1]} G(t, s)=G(1, s)$ and $\min _{t \in[0,1]} G(t, s) \geq$ $z_{5} G(s, s)=z_{5}\|G(., s)\|$.

(vi) Take $s \in\left(\xi_{m-2}, 1\right]$ and $t \leq s$. Since $G(t, s)$ is increasing in $t$ and $0<z_{6}<1$, we have $\min _{t \in[0,1]} G(t, s)=G(0, s)$ and $\min _{t \in[0,1]} G(t, s) \geq z_{6} G(s, s)=z_{6}\|G(., s)\|$.

Thus $\min _{t \in[0,1]} G(t, s) \geq z\|G(., s)\|$, where $z=\min \left\{z_{1}, z_{2}, z_{3}, z_{4}, z_{5}, z_{6}\right\}$.

Lemma 8. For $t, s \in[0,1]$, we have $0 \leq H(t, s) \leq H(1, s)$.

Proof. From (2.6), we obtain $0 \leq H(t, s)$. Now we will show that $H(t, s) \leq H(1, s)$.

(i) Let $t \leq s . H(t, s)=\frac{(1-s)^{\sigma-2} t^{\sigma-1}}{\Gamma(\sigma)} \leq \frac{(1-s)^{\sigma-2}}{\Gamma(\sigma)}=H(1, s)$.

(ii) Let $t \geq s$. Since $H(t, s)$ is increasing in $t$, we have $H(t, s) \leq H(1, s)$.

Lemma 9. $\min _{t \in\left[\xi_{m-2}, 1\right]} H(t, s) \geq k^{\sigma-1} H(1, s)$ for $0 \leq t, s \leq 1$, where $k \in\left(0, \xi_{m-2}\right)$ is $a$ constant.

Proof. $\quad$ (i) Take $t \leq s$. Since $H(t, s)$ is an increasing function, we get

$$
\min _{t \in\left[\xi_{m-2}, 1\right]} H(t, s)=\frac{(1-s)^{\sigma-2} \xi_{m-2}^{\sigma-1}}{\Gamma(\sigma)} \geq \frac{(1-s)^{\sigma-2}(k)^{\sigma-1}}{\Gamma(\sigma)}=k^{\sigma-1} H(1, s) .
$$

(ii) For $s \leq t$, we have

$$
\begin{aligned}
\min _{t \in\left[\xi_{m-2}, 1\right]} H(t, s) & =\frac{(1-s)^{\sigma-2} \xi_{m-2}^{\sigma-1}-\left(\xi_{m-2}-s\right)^{\sigma-1}}{\Gamma(\sigma)} \\
& >\frac{(1-s)^{\sigma-2} \xi_{m-2}^{\sigma-1}-\left(\xi_{m-2}-\xi_{m-2} s\right)^{\sigma-1}}{\Gamma(\sigma)} \\
& =\frac{\xi_{m-2}^{\sigma-1}\left((1-s)^{\sigma-2}-(1-s)^{\sigma-1}\right)}{\Gamma(\sigma)} \\
& =\xi_{m-2}^{\sigma-1} H(1, s) \\
& >k^{\sigma-1} H(1, s) .
\end{aligned}
$$

Thus $\min _{t \in\left[\xi_{m-2}, 1\right]} H(t, s) \geq k^{\sigma-1} H(1, s)$. 
From Lemma 4 and Lemma 5, we know that $u(t)$ is a solution of the problem (1.1) if and only if

$$
u(t)=\int_{0}^{1} G(t, s) \int_{0}^{1} H(s, \tau) f(\tau, u(\tau)) d \tau d s .
$$

Let $E$ denote the Banach space $C[0,1]$ with the norm $\|u\|=\max _{t \in[0,1]}|u(t)|$. Define the cone $P \subset E$ by

$$
P=\left\{u \in E: u(t) \geq 0, \min _{t \in[0,1]} u(t) \geq z\|u\|\right\}
$$

where $z$ is given in (2.11).

We can define the operator $A: P \rightarrow E$ by

$$
A u(t)=\int_{0}^{1} G(t, s) \int_{0}^{1} H(s, \tau) f(\tau, u(\tau)) d \tau d s,
$$

where $u \in P$. Therefore solving (2.12) in $P$ is equivalent to finding fixed points of the operator $A$.

Lemma 10. If the conditions $(\mathrm{H} 1)$ and $(\mathrm{H} 2)$ hold, then $A P \subset P$.

Proof. If $u \in P$, then $A u(t) \geq 0$ on $[0,1]$ by using Lemma 6 and Lemma 8. On the other hand, we have

$$
\begin{aligned}
\min _{t \in[0,1]} A u(t) & =\int_{0}^{1} \min _{t \in[0,1]} G(t, s) \int_{0}^{1} H(s, \tau) f(\tau, u(\tau)) d \tau d s \\
& \geq z \int_{0}^{1} \max _{t \in[0,1]} G(t, s) \int_{0}^{1} H(s, \tau) f(\tau, u(\tau)) d \tau d s \\
& =z\|A u\|,
\end{aligned}
$$

by Lemma 7. Thus $A u \in P$ and therefore $A P \subset P$.

In order to follow the main results of this paper easily, now we state the fixed point theorems which we applied to prove main theorems.

Theorem 1 (Krasnosel'skii Fixed Point Theorem, [12]). Let $E$ be a Banach space, and let $K \subset E$ be a cone. Assume $\Omega_{1}$ and $\Omega_{2}$ are open bounded subsets of $E$ with $0 \in \Omega_{1}, \overline{\Omega_{1}} \subset \Omega_{2}$, and let

$$
A: K \cap\left(\overline{\Omega_{2}} \backslash \Omega_{1}\right) \rightarrow K
$$

be a completely continuous operator such that either

(i) $\|A u\| \leq\|u\|$ for $u \in K \cap \partial \Omega_{1},\|A u\| \geq\|u\|$ for $u \in K \cap \partial \Omega_{2}$ or

(ii) $\|A u\| \geq\|u\|$ for $u \in K \cap \partial \Omega_{1},\|A u\| \leq\|u\|$ for $u \in K \cap \partial \Omega_{2}$

hold. Then $A$ has a fixed point in $K \cap\left(\overline{\Omega_{2}} \backslash \Omega_{1}\right)$. 
Theorem 2 (Leggett-Williams Fixed Point Theorem, [14]). Let $P$ be a cone in the real Banach space E. Set

$$
\begin{gathered}
P_{r}:=\{x \in P:\|x\|<r\}, \\
P(\psi, a, b):=\{x \in P: a \leq \psi(x),\|x\| \leq b\} .
\end{gathered}
$$

Suppose $A: \overline{P_{r}} \rightarrow \overline{P_{r}}$ be a completely continuous operator and $\psi$ be a nonnegative continuous concave functional on $P$ with $\psi(u) \leq\|u\|$ for all $u \in \overline{P_{r}}$. If there exists $0<p<q<l \leq r$ such that the following conditions hold,

(i) $\{u \in P(\psi, q, l): \psi(u)>q\} \neq \varnothing$ and $\psi(A u)>q$ for all $u \in P(\psi, q, l)$,

(ii) $\|A u\|<p$ for $\|u\| \leq p$,

(iii) $\psi(A u)>$ q for $u \in P(\psi, q, r)$ with $\|A u\|>l$,

then $A$ has at least three fixed points $u_{1}, u_{2}$ and $u_{3}$ in $\overline{P_{r}}$ satisfying

$$
\left\|u_{1}\right\|<p, \psi\left(u_{2}\right)>q, p<\left\|u_{3}\right\| \text { with } \psi\left(u_{3}\right)<q .
$$

\section{MAin REsults}

For convenience, we introduce the following notations. Let

$$
\begin{gathered}
M=\int_{0}^{1} H(1, \tau) d \tau, \\
L=\int_{0}^{1} G(s, s) d s, \\
I=\int_{\xi_{m-2}}^{1} G(s, s) d s .
\end{gathered}
$$

Now, we will give the sufficient conditions to have at least one positive solution for the BVP (1.1). Krasnosel'skii fixed point theorem will be used to prove the next theorem.

Theorem 3. Suppose (H1) and (H2) hold. In addition, let there exist numbers $0<r<R<\infty$ such that the function $f$ satisfies the following conditions:

(i) $f(t, u)<\frac{1}{L M} u(t)$ for $(t, u) \in[0,1] \times[0, r]$,

(ii) $f(t, u)>\frac{1}{k^{\sigma-1} z^{2} I M} u(t)$ for $(t, u) \in[0,1] \times[R, \infty)$.

Then the BVP (1.1) has at least one positive solution.

Proof. Define the open bounded subsets of $E$ by $\Omega_{1}=\{u \in P:\|u\|<r\}$ and $\Omega_{2}=\left\{u \in P:\|u\|<\frac{R}{z}\right\}$. It is easy to check that $A: P \cap\left(\overline{\Omega_{2}} \backslash \Omega_{1}\right) \rightarrow P$ is completely continuous operator. 
If $u \in P \cap \partial \Omega_{1}$, then $\|u\|=r$. Therefore, by using the hypothesis $(i)$, Lemma 6 and Lemma 8 ,

$$
\begin{aligned}
A u(t) & =\int_{0}^{1} G(t, s) \int_{0}^{1} H(s, \tau) f(\tau, u(\tau)) d \tau d s \\
& <\frac{1}{L M} \int_{0}^{1} G(t, s) \int_{0}^{1} H(s, \tau) u(\tau) d \tau d s \\
& \leq \frac{1}{L M}\|u\| \int_{0}^{1} G(t, s) \int_{0}^{1} H(s, \tau) d \tau d s \\
& \leq \frac{1}{L M}\|u\| \int_{0}^{1} G(s, s) \int_{0}^{1} H(1, \tau) d \tau d s \\
& =\|u\|,
\end{aligned}
$$

for all $t \in[0,1]$. Thus $\|A u\| \leq\|u\|$ for $u \in P \cap \partial \Omega_{1}$. On the other hand, $u \in P \cap \partial \Omega_{2}$ implies

$$
u(t) \geq z\|u\|=R
$$

for $t \in[0,1]$ and

$$
\begin{aligned}
A u(t) & =\int_{0}^{1} G(t, s) \int_{0}^{1} H(s, \tau) f(\tau, u(\tau)) d \tau d s \\
& >\frac{1}{k^{\sigma-1} z^{2} I M} \int_{0}^{1} G(t, s) \int_{0}^{1} H(s, \tau) u(\tau) d \tau d s \\
& \geq \frac{1}{k^{\sigma-1} z^{2} I M} z\|u\| \int_{0}^{1} G(t, s) \int_{0}^{1} H(s, \tau) d \tau d s \\
& \geq \frac{1}{k^{\sigma-1} z^{2} I M} z\|u\| z k^{\sigma-1} \int_{\xi_{m-2}}^{1} G(s, s) \int_{0}^{1} H(1, \tau) d \tau d s \\
& =\|u\|,
\end{aligned}
$$

from (ii), Lemma 7 and Lemma 9. Consequently, $\|A u\| \geq\|u\|$ for $u \in P \cap \partial \Omega_{2}$.

By the first part of Theorem 1, $A$ has a fixed point in $P \cap\left(\overline{\Omega_{2}} \backslash \Omega_{1}\right)$, such that $r \leq\|u\| \leq \frac{R}{z}$. Therefore BVP (1.1) has at least one positive solution. 
Now we will use the Legget-Williams fixed point theorem to prove the next theorem.

Theorem 4. Assume that $(\mathrm{H} 1)$ and $(\mathrm{H} 2)$ hold. Suppose that there exist numbers $0<p<q<\frac{q}{z} \leq r$ such that the function $f$ satisfies the following conditions:

(i) $f(t, u) \leq \frac{r}{M L}$ for $(t, u) \in[0,1] \times[0, r]$,

(ii) $f(t, u)<\frac{p}{M L}$ for $(t, u) \in[0,1] \times[0, p]$,

(iii) $f(t, u)>\frac{q}{k^{\sigma-1} z I M}$ for $(t, u) \in[0,1] \times\left[q, \frac{q}{z}\right]$,

where $z, M, L$ and I are as in (2.11), (3.1), (3.2) and (3.3), respectively and $k$ is defined in Lemma 9. Then the BVP (1.1) has at least three positive solutions $u_{1}, u_{2}$ and $u_{3}$ satisfying

$$
\begin{array}{r}
\max _{t \in[0,1]} u_{1}(t)<p, \min _{t \in[0,1]} u_{2}(t)>q \\
\max _{t \in[0,1]} u_{3}(t)>p \text { with } \min _{t \in[0,1]} u_{3}(t)<q .
\end{array}
$$

Proof. Define the nonnegative, continuous, concave functional $\psi: P \rightarrow[0, \infty)$ to be $\psi(y)=\min _{t \in[0,1]} u(t)$ and the cone $P$ as in (2.13). For all $u \in P$, we have $\psi(u) \leq\|u\|$. Now we show that $A: \overline{P_{r}} \rightarrow \overline{P_{r}}$ is completely continuous operator. If $u \in \overline{P_{r}}$, then $0 \leq u(t) \leq r$ for all $t \in[0,1]$. We get,

$$
\begin{aligned}
\|A u\| & =\max _{t \in[0,1]}|A u(t)| \\
& =\int_{0}^{1} \max _{t \in[0,1]} G(t, s) \int_{0}^{1} H(s, \tau) f(\tau, u(\tau)) d \tau d s \\
& \leq \int_{0}^{1} G(s, s) \int_{0}^{1} H(1, \tau) f(\tau, u(\tau)) d \tau d s \\
& \leq \frac{r}{M L} \int_{0}^{1} G(s, s) \int_{0}^{1} H(1, \tau) d \tau d s \\
& \leq r
\end{aligned}
$$

by hypothesis (i), Lemma 6 and Lemma 8 . Thus $A: \overline{P_{r}} \rightarrow \overline{P_{r}}$. It easy to check that $A: \overline{P_{r}} \rightarrow \overline{P_{r}}$ is completely continuous.

Since $z<1, u(t)=\frac{q}{z} \in P\left(\psi, q, \frac{q}{z}\right)$ and $\psi\left(\frac{q}{z}\right)>q$. Then, we have

$$
\left\{u \in P\left(\psi, q, \frac{q}{z}\right): \psi(u)>q\right\} \neq \varnothing .
$$


On the other hand, for all $u \in P\left(\psi, q, \frac{q}{z}\right)$, we have $q \leq u(t) \leq \frac{q}{z}$ for $t \in[0,1]$. Using assumption (iii), Lemma 7 and Lemma 9 , we find

$$
\begin{aligned}
\psi(A u) & =\min _{t \in[0,1]} \int_{0}^{1} G(t, s) \int_{0}^{1} H(s, \tau) f(\tau, u(\tau)) d \tau d s \\
& \geq z \int_{0}^{1} G(s, s) \int_{0}^{1} H(s, \tau) f(\tau, u(\tau)) d \tau d s \\
& \geq z \int_{\xi_{m-2}}^{1} G(s, s) \int_{0}^{1} H(s, \tau) f(\tau, u(\tau)) d \tau d s \\
& \geq k^{\sigma-1} z \int_{\xi_{m-2}}^{1} G(s, s) \int_{0}^{1} H(1, \tau) f(\tau, u(\tau)) d \tau d s \\
& \geq k^{\sigma-1} z \frac{q}{k^{\sigma-1} z I M} \int_{\xi_{m-2}}^{1} G(s, s) \int_{0}^{1} H(1, \tau) d \tau d s=q .
\end{aligned}
$$

Thus condition (i) of Theorem 2 holds.

For $\|u\|<p$, we have $0 \leq u(t) \leq p$ for $t \in[0,1]$. Then from assumption (ii), Lemma 6 and Lemma 8, we obtain

$$
\begin{aligned}
\|A u\| & =\max _{t \in[0,1]} \int_{0}^{1} G(t, s) \int_{0}^{1} H(s, \tau) f(\tau, u(\tau)) d \tau d s \\
& \leq \int_{0}^{1} G(s, s) \int_{0}^{1} H(1, \tau) f(\tau, u(\tau)) d \tau d s \\
& <\frac{p}{M L} \int_{0}^{1} G(s, s) \int_{0}^{1} H(1, \tau) d \tau d s=p .
\end{aligned}
$$

It follows that condition (ii) of Theorem 2 is satisfied.

Finally, we will check condition (iii) of Theorem 2. We suppose that $u \in P(\psi, q, r)$ with $\|A u\|>\frac{q}{z}$. Then we obtain

$$
\psi(A u)=\min _{t \in[0,1]} A u(t) \geq z\|A u\|>q .
$$

Since all conditions of the Legget-Williams fixed point theorem are satisfied, the BVP (1.1) has at least three positive solutions $u_{1}, u_{2}$ and $u_{3}$ such that

$$
\max _{t \in[0,1]} u_{1}(t)<p, \min _{t \in[0,1]} u_{2}(t)>q
$$




$$
\max _{t \in[0,1]} u_{3}(t)>p \text { with } \min _{t \in[0,1]} u_{3}(t)<q .
$$

Example 1. Taking $n=5, m=3, \xi_{1}=\frac{1}{2}, \alpha=\gamma=\delta=3, \beta=4, a_{1}=b_{1}=1, k=\frac{1}{4}$ and $\sigma=\frac{5}{2}$, we consider the following boundary value problem:

$$
\left\{\begin{array}{l}
-D_{0_{+}}^{\frac{5}{2}}\left(u^{\prime \prime}(t)\right)=\frac{100000 u^{2}}{u^{2}+1}, t \in[0,1] \\
u^{\prime \prime}(0)=u^{\prime \prime \prime}(0)=0, u^{\prime \prime \prime}(1)=0 \\
3 u(0)-4 u^{\prime}(0)=u^{\prime}\left(\frac{1}{2}\right) \\
3 u(1)+3 y^{\prime}(1)=u^{\prime}\left(\frac{1}{2}\right) .
\end{array}\right.
$$

Then we get $K=30, M=\frac{16}{45 \sqrt{\pi}}, L=\frac{47}{60}, I=0,45, z=\frac{3}{35}$ and $k^{\sigma-1}=k^{\frac{3}{2}}=0.125$.

If we take $p=7.10^{-7}, q=10$ and $r=130000$, then $0<p<q<\frac{q}{z}<r$ and all the conditions in Theorem 4 are fulfilled. Hence, by Theorem 4, the BVP (3.5) has at least three positive solutions $u_{1}, u_{2}$ and $u_{3}$ satisfying

$$
\begin{array}{r}
\max _{t \in[0,1]} u_{1}(t)<p, \min _{t \in[0,1]} u_{2}(t)>q \\
\max _{t \in[0,1]} u_{3}(t)>p \text { with } \min _{t \in[0,1]} u_{3}(t)<q .
\end{array}
$$

\section{REFERENCES}

[1] M. I. Abbas and M. A. Ragusa, "Solvability of Langevin equations with two Hadamard fractional derivatives via Mittag-Leffler functions.” arXiv:2006.07608, pp. 1-17, 2020.

[2] R. P. Agarwal, S. Gala, and M. A. Ragusa, "A regularity criterion in weak spaces to Boussinesq equations." Mathematics, vol. 8, no. 6, pp. 1-11, 2020, doi: 10.3390/math8060920.

[3] B. Ahmad, D. Garout, S. K. Ntouyas, and A. Alsaedi, "Caputo fractional differential inclusions of arbitrary order with nonlocal integro-multipoint boundary conditions." Miskolc Math. Notes, vol. 20, no. 2, pp. 683-699, 2019, doi: 10.18514/MMN.2019.2241.

[4] B. Ahmad and J. J. Nieto, "Existence of solutions for nonlocal boundary value problems of higherorder nonlinear fractional differential equations." Abstr. Appl. Anal., vol. 2009, Article ID 494720, pp. 1-9, 2009, doi: 10.1155/2009/494720.

[5] B. Ahmad and S. K. Ntouyas, "A note on fractional differential equations with fractional separated boundary value conditions.” Abstr. Appl. Anal., vol. 2012, Article ID 818703, pp. 1-11, 2012, doi: $10.1155 / 2012 / 818703$.

[6] S. Benbernou, S. Gala, and M. A. Ragusa, "On the regularity criteria for the 3D magnetohydrodynamic equations via two components in terms of BMO space." Math. Meth. Appl. Sci., vol. 37, no. 15, pp. 2320-2325, 2014, doi: 10.1002/mma.2981.

[7] A. Borhanifar, M. A. Ragusa, and S. Valizadehaz, "High-order numerical method for twodimensional Riesz space fractional advection-dispersion equation." arXiv:2006.04111, pp. 1-18, 2020 . 
[8] M. El-Shahed, "Positive Solutions for Boundary Value Problem of Nonlinear Fractional Differential Equation." Abstr. Appl. Anal., vol. 2007, Article ID 10368, pp. 1-8, 2007, doi: $10.1155 / 2007 / 10368$.

[9] F. T. Fen, I. Y. Karaca, and O. B. Ozen, "Positive solutions of boundary value problems for pLaplacian fractional differential equations." Filomat, vol. 31, no. 5, pp. 1265-1277, 2017, doi: 10.2298/FIL1705265F.

[10] K. Ghanbari and Y. Gholami, "Existence and multiplicity of positive solutions for $m$-point nonlinear fractional differential equations on the half line." Electron. J. Differential Equations, vol. 2012, no. 238, pp. 1-15, 2012.

[11] M. Günendi and I. Yaslan, "Positive solutions of higher-order nonlinear multi-point fractional equations with integral boundary conditions." Fract. Calc. Appl. Anal., vol. 19, no. 4, pp. 9891009, 2016, doi: 10.1515/fca-2016-0054.

[12] D. Guo and V. Lakshmikantham, Nonlinear Problems in Abstract Cones. San Diego: Academic Press, 1988.

[13] A. A. Kilbas, H. M. Srivastava, and J. J. Trujillo, Theory and Applications of Fractional Differential Equations. Amsterdam: Elsevier, 2006.

[14] R. W. Leggett and L. R. Williams, "Multiple positive fixed points of nonlinear operators on ordered Banach space.” Indiana Univ. Math. J., vol. 28, no. 4, pp. 673-688, 1979.

[15] S. Liang and J. Zhang, "Existence of multiple positive solutions for $m$-point fractional boundary value problems on an infinite interval." Math. Comput. Modelling, vol. 54, no. 5-6, pp. 1334 1346, 2011, doi: 10.1016/j.mcm.2011.04.004.

[16] N. Nyamoradi and M. Javidi, "Positive solutions for fractional differential equations with $p$ Laplacian." J. Nonlinear Anal. Optim., vol. 3, no. 2, pp. 239-253, 2012.

[17] K. Oldham and J. Spanier, Fractional Calculus: Theory and Applications, Differentiation and Integration to Arbitrary Order. New York: Academic Press, 1974.

[18] M. A. Ragusa, "Necessary and sufficient condition for a VMO function." Applied Math. Comput., vol. 218, no. 24, pp. 11952-11958, 2012, doi: 10.1016/j.amc.2012.06.005.

[19] M. A. Ragusa and A. Razani, "Weak solutions for a system of quasilinear elliptic equations." Contrib. Math. (Shahin Digital Publisher), vol. 1, pp. 11-16, 2020, doi: 10.47443/cm.2020.0008.

[20] J. Sabatier, O. P. Agrawal, and J. A. T. Machado, Advances in Fractional Calculus. Dordrecht: Springer, 2007.

[21] S. G. Samko, A. A. Kilbas, and O. I. Marichev, Fractional Integral and Derivatives: Theory and Applications. Yverdon: Gordon and Breach, 1993.

[22] X. Su and S. Zhang, "Solutions to boundary-value problems for nonlinear differential equations of fractional order.” Electron. J. Differential Equations, vol. 2009, no. 26, pp. 1-15, 2009.

[23] W. Zhong, "Positive solutions for multipont boundary value problem of fractional differential equations." Abstr. Appl. Anal., vol. 2010, no. 601492, pp. 1-15, 2010, doi: 10.1155/2010/601492.

Authors' addresses

İsmail Yaslan

(Corresponding author) Pamukkale University, Department of Mathematics, 20070 Denizli, Turkey

E-mail address: iyaslan@pau.edu.tr

Mustafa Günendi

Pamukkale University, Department of Mathematics, 20070 Denizli, Turkey

E-mail address: mustafa_87875@hotmail.com 